\title{
Numerical Aero-Thermal Study of High-Pressure Turbine Nozzle Guide Vane: Effects of Inflow Conditions ${ }^{\text {a) }}$
}

\author{
H. M. Phan, ${ }^{1, b)}$ P. H. Duan, ${ }^{1}$ and C. T. Dinh ${ }^{2}$ \\ ${ }^{1}$ Department of Engineering Science, University of Oxford, Oxford, OX2 OES, United Kingdom \\ ${ }^{2}$ School of Transportation Engineering, Hanoi University of Science and Technology, Hanoi, Vietnam
}

Accurate predictability of high-pressure turbine nozzle guide vane aero-thermal performance is highly desired in the development campaign due to the exposure of the component to a frequent and high heat load. In this paper, the representative vane profile in modern aero-engines is numerically studied. Aerodynamics and aero-thermal validations of the blade profile have been performed in comparison with the available experimental data. It has been showed that a satisfactory agreement could be achieved with the use of the transitional turbulence model SST $\gamma-\theta$ due to its superiority in capturing the laminarturbulent transition. Sensitivity studies to the increase in inlet turbulence intensity, inlet endwall boundary layer thickness, and inlet total temperature profile have been performed to understand the impact of inflow conditions uncertainty on the aerothermal predictability. Increasing the inlet turbulence intensity increases the pressure surface heat transfer coefficient and induces an earlier transition onset on the suction surface. Due to the rapid decay of turbulence intensity in the numerical model, the use of an artificially high inlet turbulence intensity has been shown to be effective in the prediction improvement. On the other hand, the change in inlet boundary layer thickness influences the formation and strength of the secondary flow, namely horseshoe vortex and passage vortex. These secondary flow phenomena affect the local blade surface heat transfer coefficient in the near-endwall region although the most significant rise in heat transfer is found on the endwall. The temperature distortion amplitude of hot streak and its relative clocking position with the vane significantly affect the heat flux distribution. In contrast, the heat transfer coefficient is less sensitive to the change in hot streak conditions. However, it has been shown that increasing the temperature distortion amplitude could induce a larger difference among different clocking configurations. In addition, decreasing the difference between the fluid and wall temperature would delay the transition onset and stabilize the boundary layer. Further analysis of the unsteadiness effects has been carried out by comparing the steady and time-averaged flow solutions. It has been observed that the discrepancy between these solutions is attributed to the flow field nonlinearity. Thus, a significant discrepancy can be found in the laminar-turbulent transition as well as the trailing edge region. However, since the contribution of these regions on the total area-averaged heat transfer is small, their influences on the total vane heat transfer is limited.

\footnotetext{
a) Contributed paper, published as part of the Proceedings of the $30^{\text {th }}$ International Symposium on Transport Phenomena ISTP30, Halong, Vietnam, November 2019.

b) Author to whom correspondence should be addressed. Electronic mail: hien.phan@eng.ox.ac.uk.
} 


\section{INTRODUCTION}

Improvements of modern gas turbine engine performance are highly dependent on the pursuit of increasing Turbine Entry Temperature (TET) and overall pressure ratio (OPR) of the engine cycle. While the latter is related to an increase in compressor pressure ratio, the former is associated with an increase in turbine inlet temperature. As a result, the high-pressure turbine (HPT) vanes of the first few stages after the combustor outlet have to withstand a significant amount of heat load. In modern gas turbine engines, the value of TET well exceeds the melting temperature of the vane materials. Therefore, the accurate prediction of vane aero-thermal performance is essential during the design phase. In the last few decades, Computational Fluid Dynamics (CFD) simulation tools have become popular among the industry for both design and analysis of the turbine vane components. The modeling fidelity is paramount for a good predictive capability of the component performance, which provides engineers a good insight and a possibility to make a design improvement. Direct Numerical Simulation (DNS) is unarguably the model with the highest fidelity, which comes at an extremely prohibitive simulation cost. Thus, studies involved DNS have typically been used with idealized configurations to investigate the basis physics mechanism behind a problem of interest [1-3]. Large Eddy Simulation (LES) is a more affordable model fidelity compared to DNS, which starts to gain its popularity in recent years $[4,5]$. However, the cost of LES simulation is still far away from the industrial expectation, in which several parametric simulations have to be carried out as part of an optimization cycle or sensitivity analysis. As a result, the turbine vane component design as of today still relies on the workhorse of Reynolds-Averaged Navier-Stokes (RANS) based simulations.

The aero-thermal performance of turbine vane components has been shown to be influenced by many factors. Thole and Knost [6] showed that high heat transfer at the endwalls was attributed to the formation of secondary flows. Hada et al. [7] found that the heat transfer on an endwall of a symmetric airfoil increases proportionally to $\mathrm{Re}^{0.71}$ if the leading edge diameter of the airfoil decreases. Arts et al. [8] reported that the transition was triggered upstream at high turbulence intensity, whereas the overall heat transfer coefficient increased with Reynolds number. Within the same experimental campaign, they also concluded that free-stream Mach number affected the development of the turbulent boundary layer at the rear part of the airfoil, which grew much faster in the transonic and supersonic regimes due to the shock/boundary layer interaction [8]. Stripf et al. [9] found that roughness would cause the laminar-turbulent transition to move upstream and increase turbulent heat transfer, although the heat transfer in the laminar region was unaffected.

In addition, a variety of turbomachinery predictions has been shown to be greatly influenced by the inflow boundary conditions [10-14]. From the literature, the turbine vane aero-thermal performance is also affected by the inflow conditioning. Choi et al. [15] found that increasing the inlet turbulence intensity promoted the boundary layer transition and enhanced local 
heat transfer coefficient. Zhang et al. [12] investigated the effects of inlet turbulence and endwall boundary layer on the aerothermal performance of a transonic turbine blade tip. They found that the tip surface heat transfer was insensitive to the turbulence level as well as the inlet boundary layer thickness. However, noticeable changes in heat transfer were observed for the suction side near-tip surface. Wang et al. [16] reported that the inlet total pressure profiles significantly affected the structures of both passage vortices and counter-rotating vortices, which in turn induced a significant change of the airfoil heat transfer. Because the high-pressure turbine blade rows are situated immediately downstream of the combustor, they are exposed to strong non-uniformity in velocity and temperature due to what is known as "hot streak". The impacts of the hot streak on turbine vane aero-thermal performance have been widely investigated by some researchers. He et al. [17] showed that the unsteady heat load of rotor blades was significantly changed with different wavelength of the temperature distortion, in which the strongest dependence on the relative position between the hot streak and vane was found when the hot streak wavelength was comparable to the blade pitch. Wang et al. [18] found that hot streak position not only affected the airfoil surface temperature variations but also slightly changed the vane and rotor mid-span heat transfer coefficient. Rahim and He [19] investigated the combination effects of non-uniform inlet temperature and velocity profiles. They found that the swirling flow considerably redistributed the hot fluid in the radial direction within the vane passage, which led to a more uniform rotor inlet radial temperature profile compared to the case with hot streak only. Rahim et al. [20] compared the nominal vane to its compound lean design under hot streak condition. The leaned vane was showed to have a more favorable heat flux distribution compared to the nominal configuration, which was largely affected by the change in fluid driving temperature rather than heat transfer coefficient. Khanal et al. [21] reported a significant interplay of swirl and hot streak on the aerodynamic and heat transfer characteristics. They also showed that the predicted blade heat transfer could not simply be superimposed when subjected to a combination of swirl and hot streak. Ong and Miller [22] studied the engine HPT stage with both inlet hot streak and vane coolant. They showed that the inflow turbulence intensity in the CFD calculation needs to be 'calibrated' in order to match with the experimental data. Feng et al. [23] investigated the aerodynamic and heat transfer performance of a 1.5 stage turbine. They concluded that the first-stage vane's heat flux distribution was controlled by the inlet temperature profile, but the heat transfer coefficient remained insensitive. In contrast, the second-stage vane's heat flux was affected strongly by the relative positions between the hot streak and the vanes. To mitigate the adverse effects of high heat load on the turbine vane surface and endwalls, film cooling techniques have been developed [24-27]. However, the interaction between the main flows and the coolant flows has been shown to be complicated and would not be considered in the current work.

With the background as introduced so far, there is a need to assess the predictive capability of RANS based simulations in comparison with the available experimental data. The responses of turbine vane aero-thermal performance to the variation in 
the inflow conditions should also be investigated within the framework of RANS simulations. Therefore, the paper will firstly start with a validation of the numerical predictions against the experimental results for both aerodynamic and heat transfer test cases. In the next step, the inlet boundary conditions will be varied parametrically to understand the influence of inflow conditioning on the turbine's aero-thermal performance. Within the scope of the present work, three inflow variables will be considered separately, namely inlet turbulence intensity, inlet boundary layer thickness, and inlet total temperature profile. Finally, a further analysis will be carried out analytically and numerically to examine the potential effects of unsteadiness on the heat transfer prediction.

\section{TEST CASE}

\section{A. Test case description}

The nozzle guide vane of a high-pressure turbine used in this study is a popular test case for turbomachinery aero-thermal study. The turbine nozzle guide vane geometry was designed in von Karman Institute [28]. Experiments were performed in the Light Piston Compression Tube facility CT-2, which is a stationary blow-down facility. The test rig comprised of three main parts: a five-meter and one-meter diameter cylinder, the test section and a downstream dump tank. For further information on the experimental facility and its working principle, one can refer to Ref. [28].

The turbine nozzle guide vane geometry used in the present study, which is denoted as the VKI LS89 blade, was designed and optimized for a downstream isentropic Mach number equal to 0.9. In the experiment, the blade was mounted in a linear cascade arrangement made of five profiles to minimize the effects of wind tunnel sidewalls. The main geometrical parameters of the VKI LS89 turbine nozzle guide vane are described in Table I and illustrated in Figure 1.

\begin{tabular}{lcl} 
TABLE I. Geometrical parameters of the LS 89 blade \\
\hline \hline Parameter & & Value \\
\hline Chord [mm] & $\mathrm{c}$ & 67.647 \\
Pitch [mm] & $\mathrm{g}$ & 57.5 \\
Throat [mm] & $\mathrm{o}$ & 14.930 \\
LE radius [mm] & $r_{L E}$ & 4.127 \\
TE radius [mm] & $r_{T E}$ & 0.710 \\
Stagger angle [degrees] & $\gamma$ & 55 \\
Span [mm] & $\mathrm{h}$ & 55.47 \\
\hline \hline
\end{tabular}




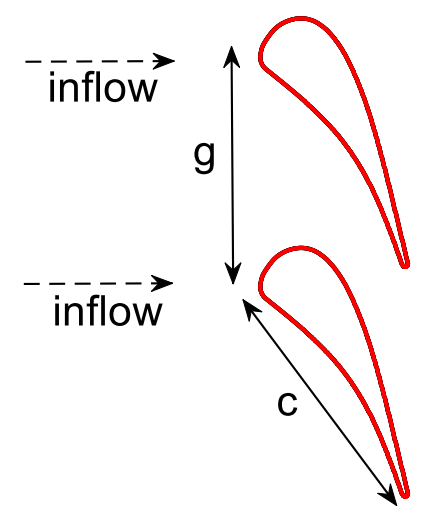

FIG. 1. The profiles of the VKI LS89 blade.

In the experimental campaign, various parameters such as Mach number, Reynolds number, and turbulence intensity had been varied such that the effect of each parameter could be investigated accordingly. Experimental data for all test case configurations are available in the public domain [29]. Within the present work, the computational results of one aerodynamics and three heat transfer test cases will be compared against experimental data for a validation purpose. Table II and Table III present the operating conditions for the aerodynamics (MUR47) and heat transfer (MUR247, MUR245, MUR239) test cases, respectively. In Table III, the experimental turbulence intensity is measured at the inlet station, whereas the reported numerical turbulence intensity is measured at the vane leading edge. The inflow numerical turbulence intensity is much higher due to the rapid decay of turbulence intensity from the inlet station to the leading edge, which will be discussed in the later part.

TABLE II. Aerodynamics experimental and numerical operating conditions - MUR47.

\begin{tabular}{ccc}
\hline Conditions & Experimental & Numerical \\
\hline Inlet flow angle $\alpha_{1}[\mathrm{deg}]$ & 0.0 & 0.0 \\
Outlet Reynolds number $R e_{2}$ & $1 \times 10^{6}$ & $1.13 \times 10^{6}$ \\
Isentropic outlet Mach number $M_{2, i s}$ & 1.0200 & 1.0201 \\
\hline \hline
\end{tabular}

TABLE III. Heat transfer experimental and numerical operating conditions - MUR247, MUR245, MUR239.

\begin{tabular}{|c|c|c|c|c|c|c|}
\hline \multirow[b]{2}{*}{ Conditions } & \multicolumn{2}{|c|}{ MUR247 } & \multicolumn{2}{|c|}{ MUR245 } & \multicolumn{2}{|c|}{ MUR239 } \\
\hline & Experimental & Numerical & Experimental & Numerical & Experimental & Numerical \\
\hline $\begin{array}{l}\text { Inlet flow angle } \alpha_{1} \text {, deg } \\
\text { Outlet Reynolds number }\end{array}$ & 0.0 & 0.0 & 0.0 & 0.0 & 0.0 & 0.0 \\
\hline $\begin{array}{l}R e_{2} \times 10^{6} \\
\text { Isentropic outlet Mach }\end{array}$ & 2.1171 & 2.4259 & 2.1343 & 2.4169 & 2.1937 & 2.4119 \\
\hline number $M_{2, i s}$ & 0.922 & 0.922 & 0.924 & 0.924 & 0.922 & 0.922 \\
\hline Turbulence intensity, $\%$ & 1.0 & 0.61 & 4.0 & 2.68 & 6.0 & 3.8 \\
\hline Inlet total temperature, $\mathrm{K}$ & 416.20 & 416.20 & 412.60 & 412.60 & 411.9 & 411.9 \\
\hline Wall temperature, $\mathrm{K}$ & 302.15 & 302.15 & 300.75 & 300.75 & 299.75 & 299.75 \\
\hline
\end{tabular}




\section{B. Data reduction}

The blade isentropic Mach number distribution is used to validate the computational aerodynamics results in comparison with the experimental data:

$$
M_{i s}=\sqrt{\frac{2}{\gamma-1}\left[\left(\frac{P_{01}}{P}\right)^{\gamma-1 / \gamma}-1\right]}
$$

where $P_{01}$ is the inlet total pressure, $P$ is the static pressure on the blade surface; $\gamma$ is the specific heat ratio.

For heat transfer test cases, the convective heat transfer coefficient is used for comparison with the experimental data:

$$
H T C=q_{w} /\left(T_{01}-T_{w}\right)
$$

where HTC is the heat transfer coefficient, $q_{w}$ is the heat flux distribution on the blade walls, $T_{01}$ is the inlet total temperature, and $T_{w}$ is the wall temperature.

For configurations with a non-uniform temperature profile (e.g. hot streak), the heat transfer coefficient shall be calculated in a slightly different way to take into account the effect of different fluid driving temperature:

$$
H T C=q_{w} /\left(T_{a w}-T_{w}\right)
$$

where $T_{a w}$ is the adiabatic wall temperature.

\section{NUMERICAL METHOD}

\section{A. Numerical model}

The commercial solver ANSYS CFX is used for the computational study. The numerical method adopted in the solver solves the unsteady Navier-Stokes equations in an iteratively implicit manner:

$$
\begin{aligned}
& \frac{\partial \rho}{\partial t}+\nabla \cdot(\rho \boldsymbol{U})=0 \\
& \frac{\partial(\rho \boldsymbol{U})}{\partial t}+\nabla \cdot(\rho \boldsymbol{U} \otimes \boldsymbol{U})=-\nabla p+\nabla \cdot \tau \\
& \frac{\partial(\rho H)}{\partial t}-\frac{\partial p}{\partial t}+\nabla \cdot(\rho \boldsymbol{U} H)=\nabla \cdot(\lambda \nabla T)+\nabla \cdot(\boldsymbol{U} \cdot \tau)
\end{aligned}
$$

The governing equations represent the conservation of mass, momentum, and energy at any time instant. For complete closure of the governing equations, the Shear Stress Transport (SST) turbulence model coupled with the $\gamma-\theta$ transitional correlation is employed [30]. The $\gamma-\theta$ transitional turbulence model has gained its popularity due to various successful applications in the aerospace and turbomachinery industries $[31,32]$

The unsteadiness effects on heat transfer have been observed in the literature, especially where there are complex interactions between the coolant and the main flow [33-35]. As no cooling feature is investigated in this study, steady 
simulations are justified to be sufficient with the assumption that the unsteady effects are small. The steady flow assumption will be later discussed in more detail in a further analysis section.

\section{B. Computational domain and boundary conditions}

In contrast to the linear cascade arrangement of five profiles in the experiment, the present computational study uses a single-passage domain. Thus, a direct periodicity is applied at the two outermost periodic interfaces, which resembles an infinite linear cascade arrangement.

The inlet boundary conditions include total pressure, total inlet temperature, flow angle, and turbulence intensity. The static pressure is used at the outlet. These boundary conditions are to be varied to match the non-dimensional parameters described in Table II and III. The blade, hub and shroud surfaces are modeled as a no-slip wall. In aerodynamic cases, an adiabatic condition is applied at the walls. In heat transfer cases with a uniform inlet temperature, a fixed isothermal wall temperature $T_{w}$ is imposed on the wall surfaces to get the heat flux $q_{w}$. On the other hand, an extra simulation at adiabatic wall condition has to be carried out for cases with a non-uniform inlet temperature profile to find the adiabatic wall temperature $T_{a w}$.

\section{Computational mesh}

Meshing is carried out in ICEM to generate an unstructured hexahedral mesh. H-O-H topology is used to create H-grid in the inlet and outlet blocks. The adjacent area of blade surfaces is modeled with O-grid to resolve the boundary layers. Figure 2 shows an O-grid mesh around the leading edge, whereas Figure 3 presents mesh distribution near the blade endwall. This mesh resolution yields about 6.2 million mesh elements for a single $3 \mathrm{D}$ one blade passage, which corresponds to $y_{\max }^{+}=0.23$ on all the wall surfaces.

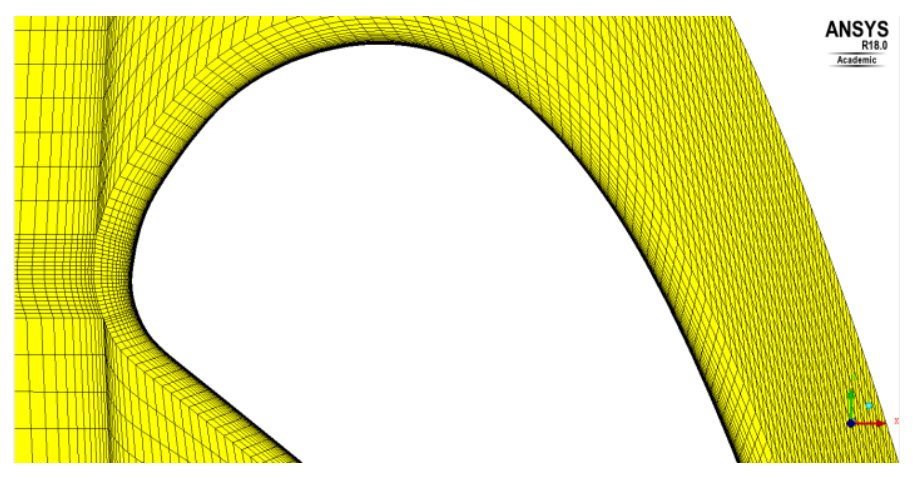

FIG. 2. O-grid mesh around the leading edge. 


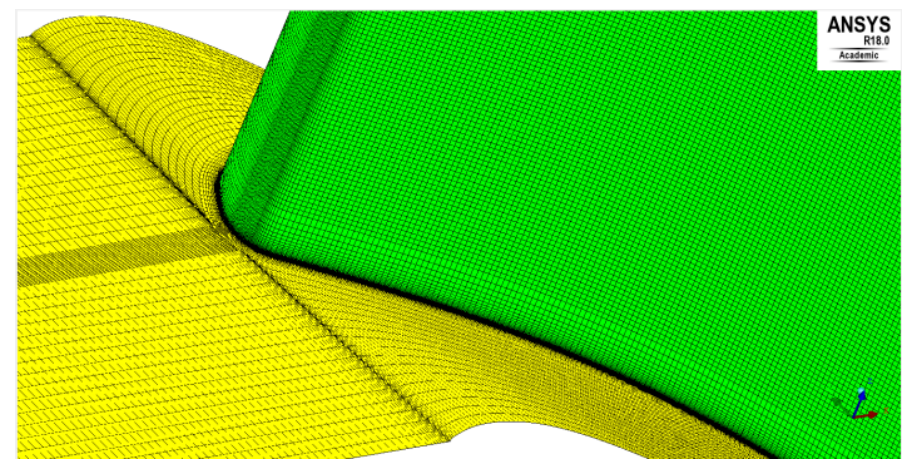

FIG. 3. Mesh near the blade endwall.

\section{VALIDATION RESULTS}

\section{A. Aerodynamics validation test case}

Figure 4 presents the isentropic Mach number on the blade surfaces predicted by the present study in comparison with the experiment [29] and previous CFD prediction in OpenFOAM [36] for the aerodynamic test case MUR47. The aft portion of the suction surface from about $60 \%$ chord to the trailing edge enters a transonic flow regime, such that $M_{i s} \approx 1-1.2$. Particularly, a shockwave is formed on the suction surface around the isentropic Mach number peak at roughly $80 \%$ chord. Both CFD simulations in the present study are able to predict the formation of shockwave, although the present study shows better agreement with the experiment on the location and the strength of the shock.

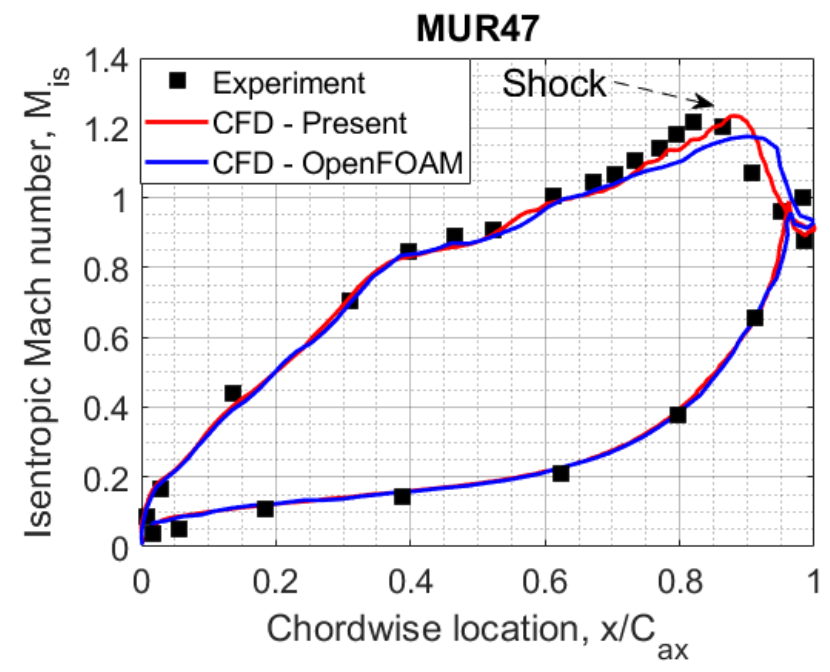

FIG. 4. Comparison of isentropic Mach number for test case MUR47.

Figure 5 presents the Mach number contour of the blade passage at mid-span for the present CFD study. The interaction between shockwaves and wakes of the adjacent blade can be observed in the CFD simulation, which is in agreement with the experimental schlieren image [28, 29]. 

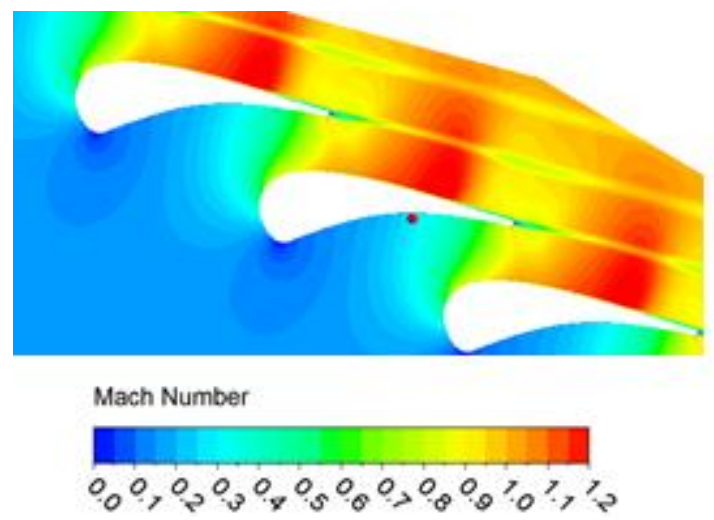

FIG. 5. Mid-span Mach number contours for test case MUR47.

\section{B. Heat transfer validation test case}

Test case MUR247 with low inlet turbulence intensity Tu is validated first against the experiment as shown in Figure 6. The heat transfer coefficient on the pressure surface is well-matched with the experimental results. On the other hand, the situation is more complicated on the suction surface due to the transitional behavior. Because of the laminar-turbulent transition on the suction surface, the use of transitional turbulence model $\gamma-\theta$ is investigated despite the extra computational cost to solve two additional transport equations of the intermittency $\gamma$ and the transition momentum thickness Reynolds number $R e_{\theta t}$. From the leading edge to about $80 \%$ chord on the suction surface, the boundary layer remains laminar with low heat transfer coefficient. From about $80 \%$ chord to the trailing edge, the boundary layer transits to turbulent, which induces a sharp increase in heat transfer coefficient. The CFD simulation with transitional turbulence model $\gamma-\theta$ gives good prediction of the suction surface transition location although the prediction of heat transfer coefficient values can only be assessed in a qualitatively manner.

Also shown in Figure 6 is the CFD results predicted by the fully turbulent and fully laminar turbulence model. If the fully turbulent model is used, a significantly erroneous prediction of surface heat transfer coefficient on both pressure and suction surfaces is observed due to the wrong specification of the boundary layer state. On the other hand, a much similar distribution of heat transfer coefficient compared to that predicted by the transitional model is observed by using the fully laminar model. The pressure surface heat transfer coefficient is predicted with an excellent match between the transitional and the fully laminar model, whereas the suction surface heat transfer coefficient is predicted with high similarity except for the rear part. Fully laminar flow predicts a plateau instead of a sharp increase of heat transfer coefficient in the rear part of the suction surface. 


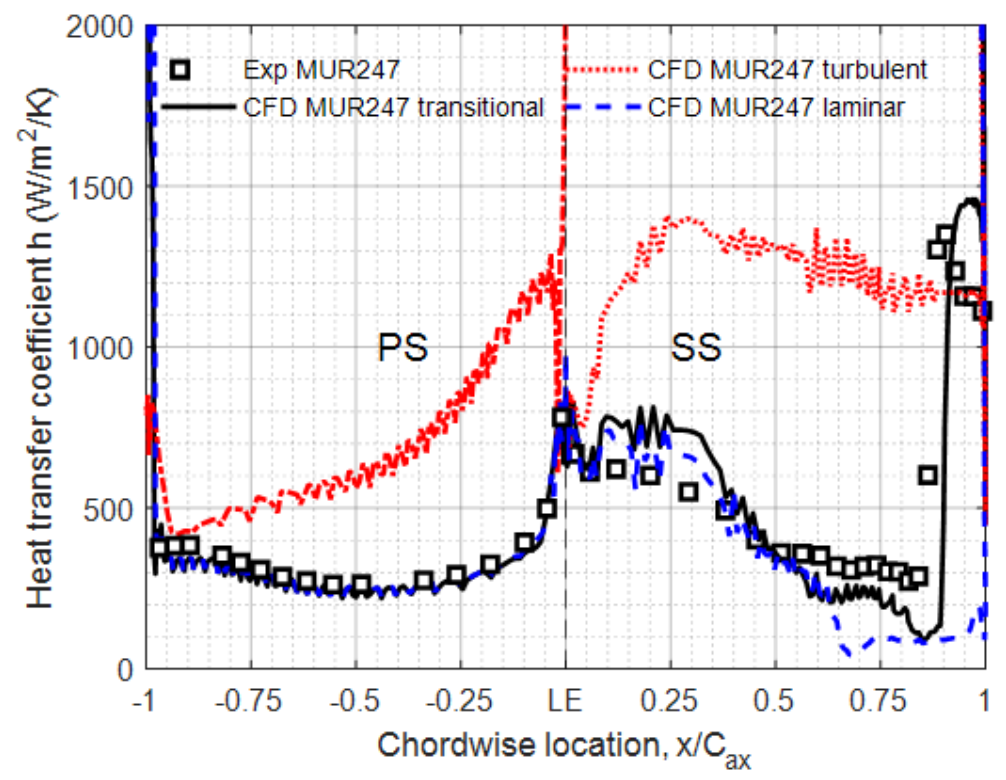

FIG. 6. Surface heat transfer coefficient distribution for test case MUR247 (transitional vs fully turbulent vs fully laminar).

In order to further examine the rear part of the suction surface, the wall shear distribution is presented in Figure 7 for the transitional, fully turbulent, and fully laminar flow model. The wall shear prediction by the fully turbulent flow is much higher than that predicted by other models. On the other hand, CFD results predicted by the transitional and fully laminar model share relatively good matching except for the rear part of the suction surface. Wall shear predicted by the laminar model has an insignificant value in this area (from about $75 \%$ chord to the trailing edge).

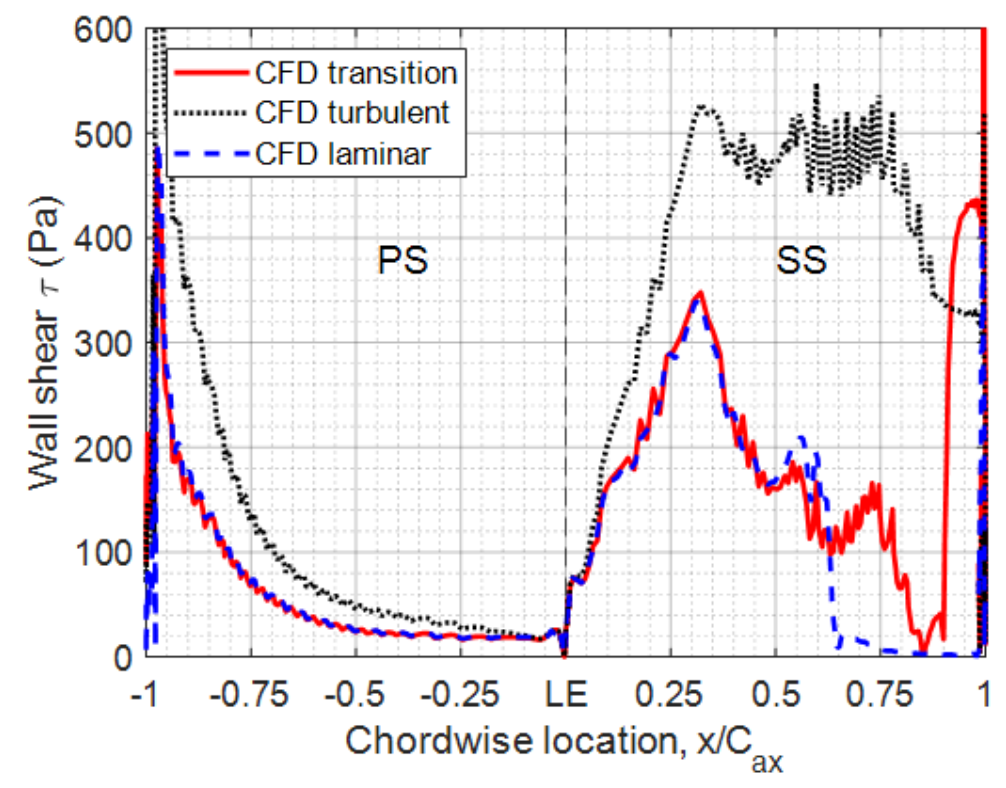

FIG. 7. Wall shear distribution of test case MUR247 (transitional vs fully turbulent vs fully laminar). 
Figure 8 shows the axial velocity contour near the blade surfaces predicted by the fully laminar model. There is a region of negative axial velocity on the rear part of the suction surface, which coincides with the region of low wall shear and low heat transfer coefficient reported in the previous discussion. This region can be regarded as the separation region of open type, in which the reattachment and subsequent transition on the surface do not take place. Thus, the boundary layer on the rear part of the suction surface remains laminar with a low heat transfer coefficient. As a result, it can be concluded that the boundary layer state has a paramount effects on the surface heat transfer coefficient. In addition, the prediction capability of the laminarturbulent transition has also been shown to be essential in capturing the correct trend in the rear part of the suction surface.

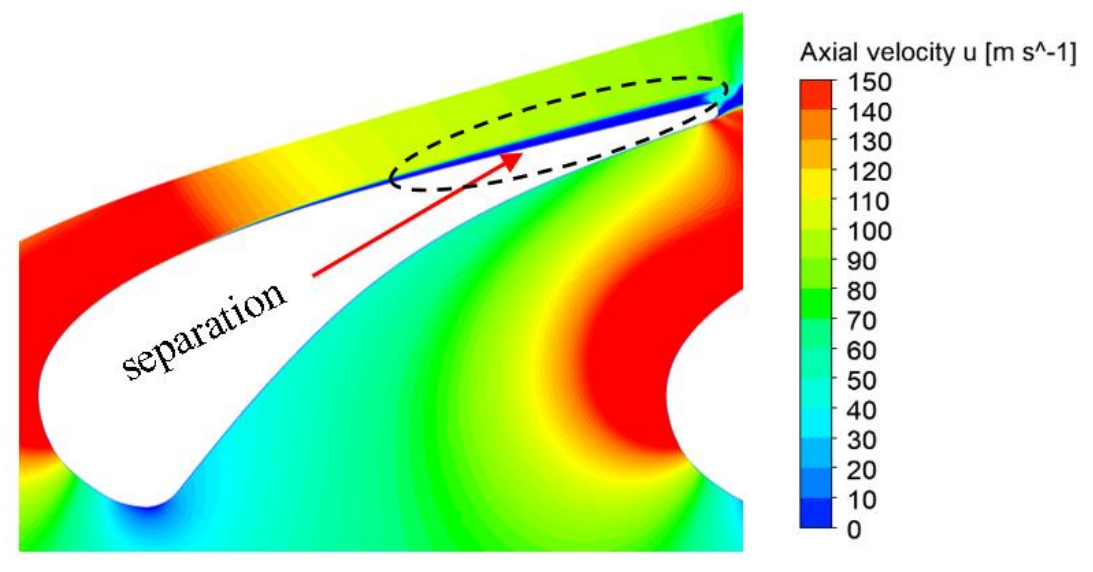

FIG. 8. Axial velocity contour of test case MUR247 predicted by the fully laminar model.

\section{EFFECTS OF INLET TURBULENCE INTENSITY}

As pointed out by Denton [10], the freestream turbulence intensity is unlikely to be known in a practical situation. However, it greatly influences the mixing of enthalpy and entropy non-uniformities, and more importantly on boundary layer transition - a key factor in the current heat transfer study as previously discussed. In this part, the inlet turbulence intensity will be varied and its effect on surface heat transfer coefficient will be assessed against the available experimental data.

Figure 9 presents the surface heat transfer coefficient obtained by the computational study and the experiment for test cases MUR247 $(T u=1 \%)$, MUR245 $(T u=4 \%)$, and MUR239 $(T u=6 \%)$. These test cases have similar outlet Reynolds and Mach number. Hence, the variations in heat transfer coefficient can be regarded as the influences of increasing turbulence intensity. 


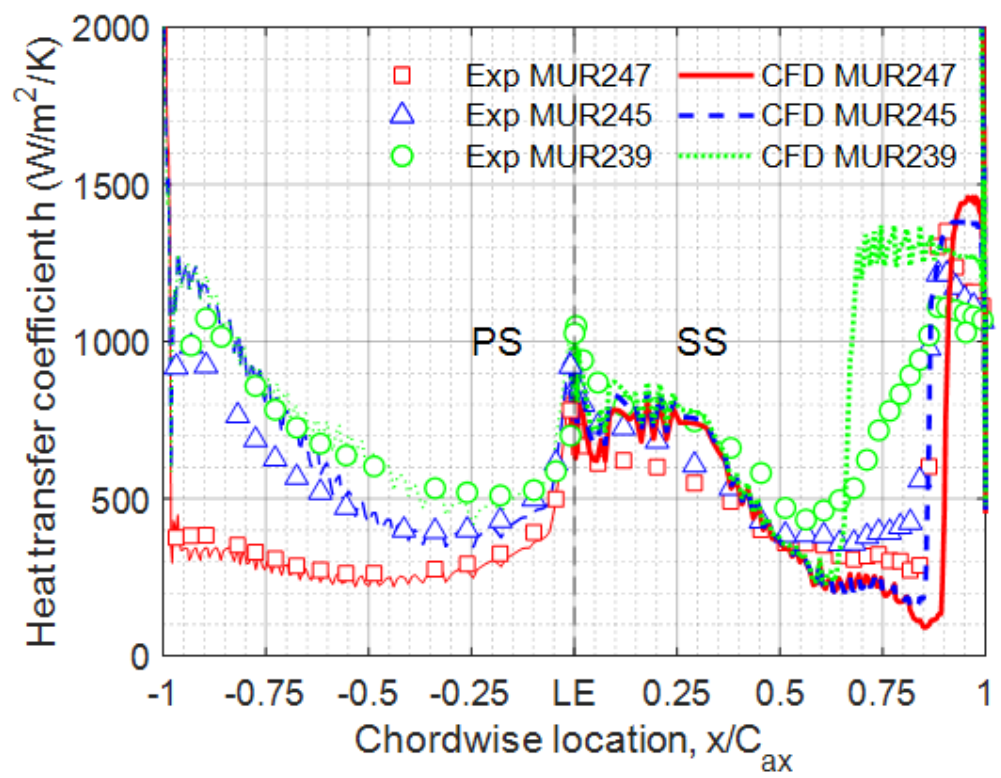

FIG. 9. Surface heat transfer coefficient for test cases with different inlet turbulent intensity.

From Figure 9, an increase in turbulence intensity would lead to an increase in heat transfer coefficient on the vast majority of the blade surfaces (e.g. MUR239 in comparison with MUR247). On the pressure surface, the heat transfer coefficient increases dramatically as the turbulence intensity increases. The response behavior can be predicted quantitatively with the current computational model. From the leading edge to the transition point on the suction surface, the heat transfer coefficient changes insignificantly in the computational model prediction, while experimental data suggest a relatively higher rise of heat transfer (although still less dramatic than that on the pressure surface). Interestingly, the transition location is well-predicted by the current model, with the trend of transition onset location moving upstream as the turbulence intensity increases, in agreement with the experimental data.

Another aspect of CFD modeling interest is that a significantly higher inlet turbulence intensity has to be used as the inlet boundary condition for test case MUR245 and MUR239 to obtain a good agreement with the experimental data. To investigate this effect, a turbulent intensity variation from the inlet station to the blade leading edge is presented in Figure 10 for test cases MUR247, MUR245, and MUR239. Comparisons with the analytical estimation can also be observed from Figure 10. The analytical estimation is obtained from Equation 7 [37]:

$$
T u=\left\{T u_{i n}^{2}\left[1+\frac{3 \cdot \rho \cdot V \cdot x \cdot \beta \cdot T u_{i n}^{2}}{2 \cdot \mu \cdot\left(\mu_{t} / \mu\right)}\right]^{-\beta^{*} / \beta}\right\}^{0.5}
$$

where $\beta=0.09$ and $\beta^{*}=0.0828$ are constants in the SST turbulence model; $\mu_{t} / \mu$ is the inlet viscosity ratio. 


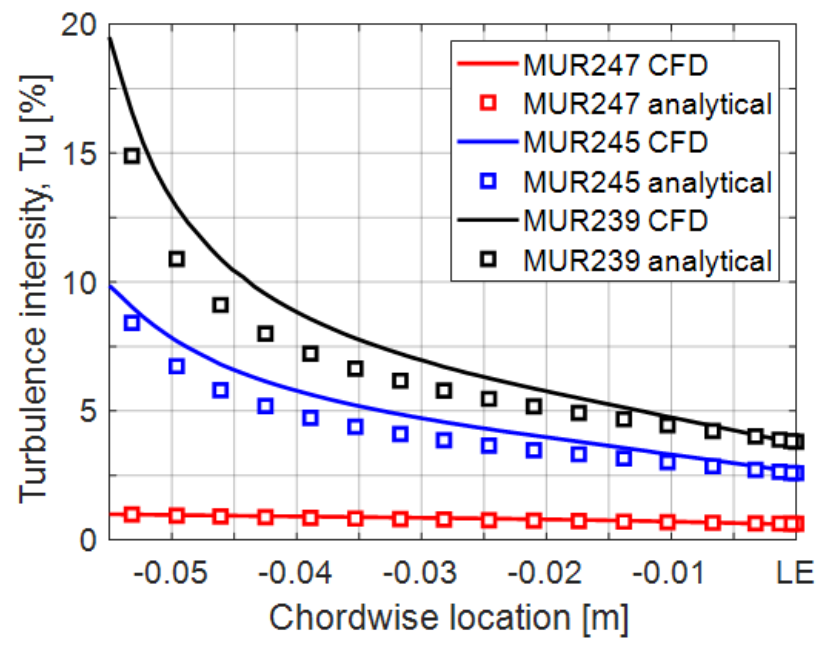

FIG. 10. Turbulence intensity decay from the inlet station to the leading edge.

It can be observed that the turbulence intensity decays exponentially from the inlet station to the leading edge in the current CFD model. The decaying rate of turbulence intensity in the experiment is not known because only the turbulence level and location at inlet station were reported. However, by using an artificially high turbulence level at the inlet, the turbulence level approaching the leading edge is comparable to that reported in the experiment.

According to Equation 7, the inlet viscosity ratio $\mu_{t} / \mu$ can also be varied to compensate for the turbulence decay effects, apart from using inlet turbulence intensity $T u_{i n}$ as in the current approach. However, it has been noticed that increasing the inlet turbulence intensity seems to be the most straightforward and effective approach for the current investigation. The modifications of inlet turbulence condition to improve heat transfer prediction are not rare. A variety of techniques have been developed for heat transfer predictability improvement, even for a high-fidelity model such as LES (e.g. synthetic turbulence generation) $[38,39]$.

\section{EFFECTS OF INLET ENDWALL BOUNDARY LAYER THICKNESS}

Inlet endwall boundary layer thickness is another factor of uncertainty [10-12]. According to Denton [10], it is perhaps the most important factor and also the least likely to be known. Inlet endwall boundary layer would have a large effect on the endwall behavior. Therefore, it is important to study the effects of inlet endwall boundary layer uncertainty on the heat transfer performance. The endwall surfaces will be modeled as uncooled, hence the coolant-flow interactions are not within the scope of the present study. The inlet endwall boundary layer thickness is varied by imposing the corresponding radial total pressure profile at the inlet. Figure 11 shows the radial inlet total pressure profile corresponding to different inlet endwall boundary layer thickness configurations investigated in this study. The deficit of total pressure near the endwall region represents the deficit of flow velocity in the boundary layer zone near the endwall. The thickness of the endwall boundary layer is given as a 
percentage of the whole blade height. The total pressure profile is radially symmetric, hence only the upper part from mid-span to the shroud is shown in Figure 11 for clarity. The remaining boundary conditions are kept constant as in test case MUR247.

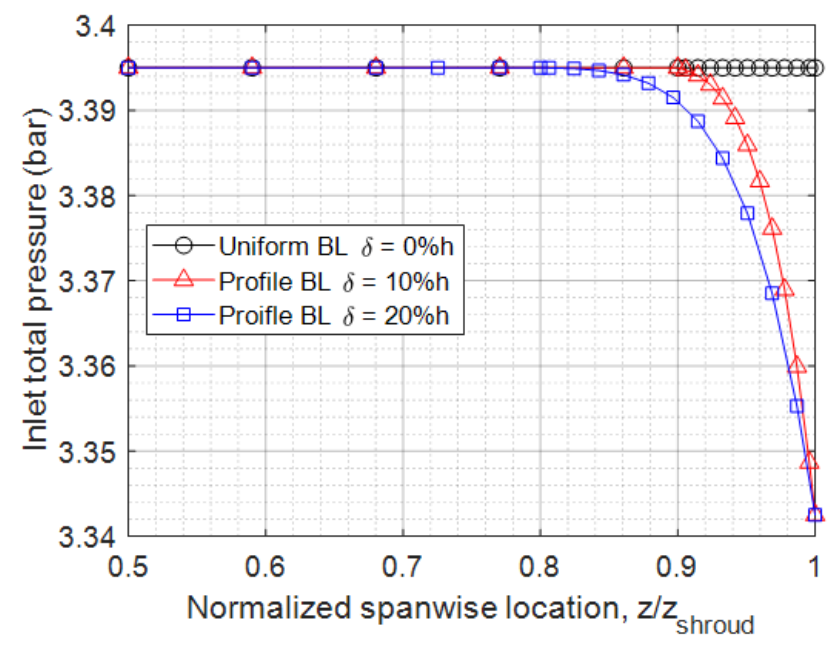

FIG. 11. Inlet total pressure profile for different inlet endwall boundary thickness configurations.

Firstly, the heat transfer coefficient on the blade suction surface is investigated for increasing inlet endwall boundary layer thickness as shown in Figure 12. The increase of inlet endwall boundary layer thickness does not affect significantly the local heat transfer distribution on the suction surface. However, certain features of three-dimensional effects are noted. The transitional behavior on the suction surface is observed at the rear part, where heat transfer increases significantly when approaching the trailing edge. The flow field near the endwalls is different from the majority of the blade span. There seems to be a disruption of the heat transfer rise in the radial direction. This is due to the formation of the corner vortex, which rolls up the blade suction surface as it travels downstream. The structure of secondary vortical flow is in agreement with that reported in the cascade environment [7]. Beneath the corner vortex, the heat transfer increases significantly.

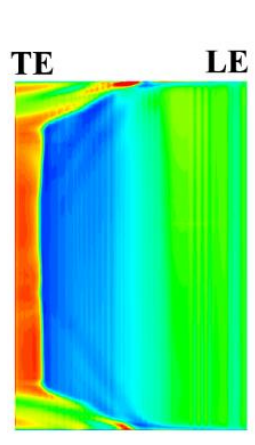

\section{Shroud}
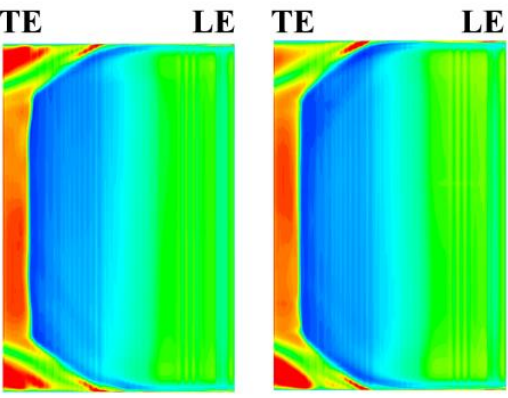

Hub
a) $\delta=0 \% h$
b) $\delta=10 \% h$
c) $\delta=20 \% h$

FIG. 12. Surface HTC contours for different inlet endwall boundary layer thickness: a) $\boldsymbol{\delta}=\mathbf{0} \% \boldsymbol{h}$, b) $\boldsymbol{\delta}=\mathbf{1 0} \% \boldsymbol{h}$, and c) $\boldsymbol{\delta}=\mathbf{2 0} \% \boldsymbol{h}$. 
The influence of the inlet endwall boundary layer thickness is greater on the endwall itself. Figure 13 shows the heat transfer coefficient contours on the hub surface for two level of inlet endwall boundary layer thickness. The area in vicinity of the blade leading edge suffers a high heat load for both configurations. The level and extension of heat transfer also increase with increasing inlet endwall boundary layer thickness. From Fig. 13b, the high heat transfer region along the travelling route of passage vortex is prominent.

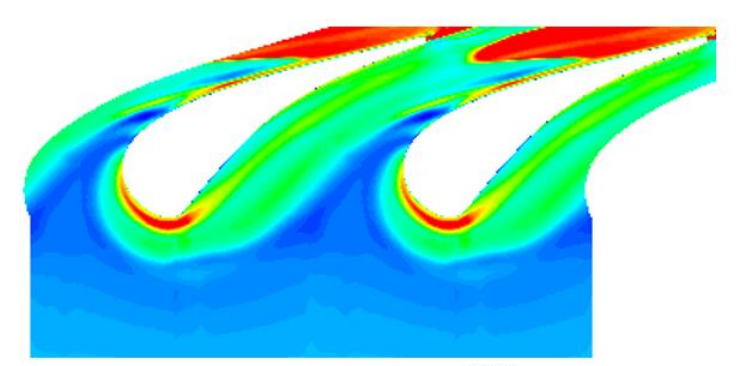

HTC

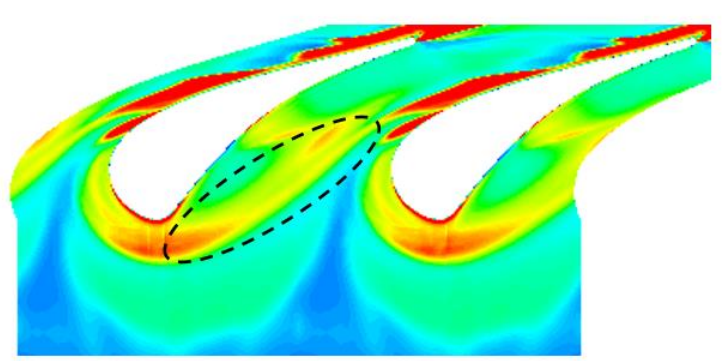

$\left[\mathrm{W} \mathrm{m}^{\wedge}-2 \mathrm{~K}^{\wedge}-1\right]$

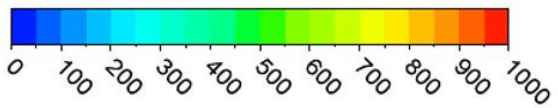
a) $\delta=0 \% \mathrm{~h}$
b) $\delta=10 \% \mathrm{~h}$

FIG. 13. HTC on the hub endwall for different inlet endwall boundary layer thickness: a) $\boldsymbol{\delta}=\mathbf{0} \% \boldsymbol{h}$, and b) $\boldsymbol{\delta}=\mathbf{1 0} \% \boldsymbol{h}$.

Figure 14 shows the iso-surface visualization by Q-criterion, which reveals the vortical structures in agreement with that reported in various literature regarding the secondary flow structure [7]. As the flow approaches the leading edge, it stagnates and separates into two counter-rotating vortices, namely the suction side horseshoe vortex and the pressure side horseshoe vortex. The pressure side horseshoe vortex is attracted by pressure gradient towards the suction leg formed around the adjacent blade. Comparing the vortical flow structure in Figure 14 with the heat transfer contour in Fig. 13b, it is apparent that the high heat transfer region is in line with the formation of these vortical structures.

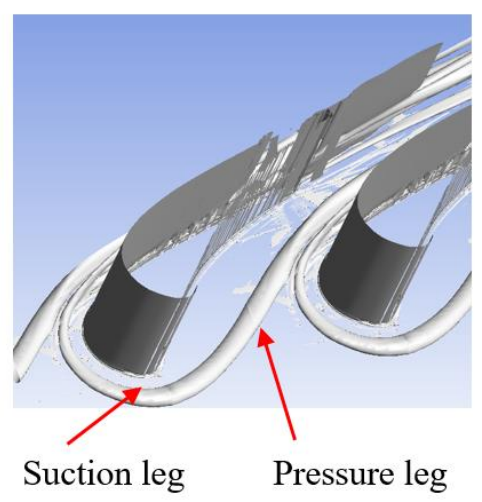

FIG. 14. Iso-surface visualization of the suction and pressure legs of passage vortex by Q-criterion. 
The area-averaged heat transfer coefficient on the hub surface is calculated based on Equation 8 to compare the overall heat transfer performance of different configurations. As the inlet endwall boundary layer thickness increases to $10 \%$ and $20 \%$, the averaged heat transfer coefficient increases by $8.2 \%$ and $13.8 \%$, respectively. Although the percentage increase seems not to be high, it should be noted that the component life span is halved for each increase of $10 \mathrm{~K}$. Undoubtedly, the current calculated increase of heat transfer coefficient would lead to premature thermal failure of the engine components.

$$
H T C_{\text {avg }}=\int H T C d A / A
$$

\section{EFFECTS OF INLET TOTAL TEMPERATURE PROFILE}

In this section, the effects of non-uniform inlet temperature distribution (hot streak) are investigated. The temperature distortion arises from the complex interaction between the combustion process and the combustor liner cooling techniques. Test case MUR239 is a baseline configuration in this section. Only the total temperature is varied, and the remaining boundary conditions are the same to the baseline case. The inlet temperature distortion is imposed as an idealized variation of total temperature across the blade pitch.

$$
T_{01, \text { hot streak }}=T_{01, \text { uniform }}+\Delta T_{01} \sin (\theta)
$$

where $T_{01 \text {,hot streak }}$ is the non-uniform inlet total temperature; $T_{01, \text { uniform }}$ is the uniform inlet total temperature, similar to the baseline case; $\Delta T_{01}$ is the total temperature distortion amplitude; $\theta$ is the circumferential position.

Three temperature distortion amplitudes $\Delta T_{01}$ will be considered: $5 \%, 10 \%$, and $15 \%$ of the nominal value of $T_{01, u n i f o r m}$ in the baseline case. Regarding the circumferential position $\theta$, several values will be investigated to further examine the effects of clocking between the hot streak and the turbine vane. There are two particular clocking positions that were extensively used in the majority of hot streak study, i.e. passage center and leading edge impingement. In the current work, $\theta=90^{\circ}$ denotes the passage center case, where the hot streak peak temperature passes through the blade passage and the cold fluid stream impinges on the blade leading edge. On the other hand, $\theta=270^{\circ}$ denotes the leading edge impingement case, where the hot fluid stream impinges on the leading edge. These two cases represent the two most extreme scenarios, where the blade is exposed to the hottest and coldest fluid flow as a result of the inlet total temperature distortion. Figure 15 presents the total temperature contours for the two particular cases of hot streak-vane relative circumferential positions. 


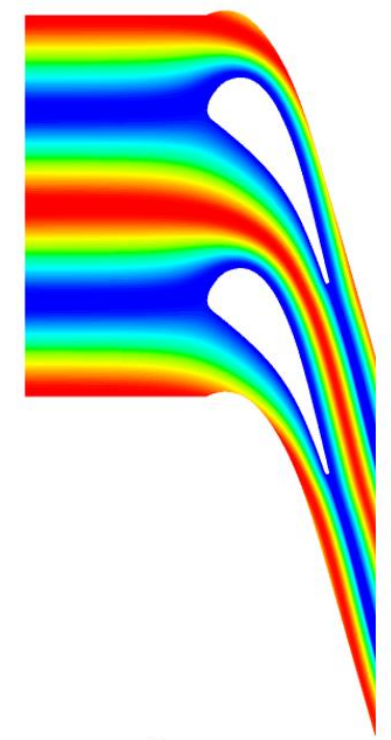

a) $\theta=90^{\circ}-$ Passage center

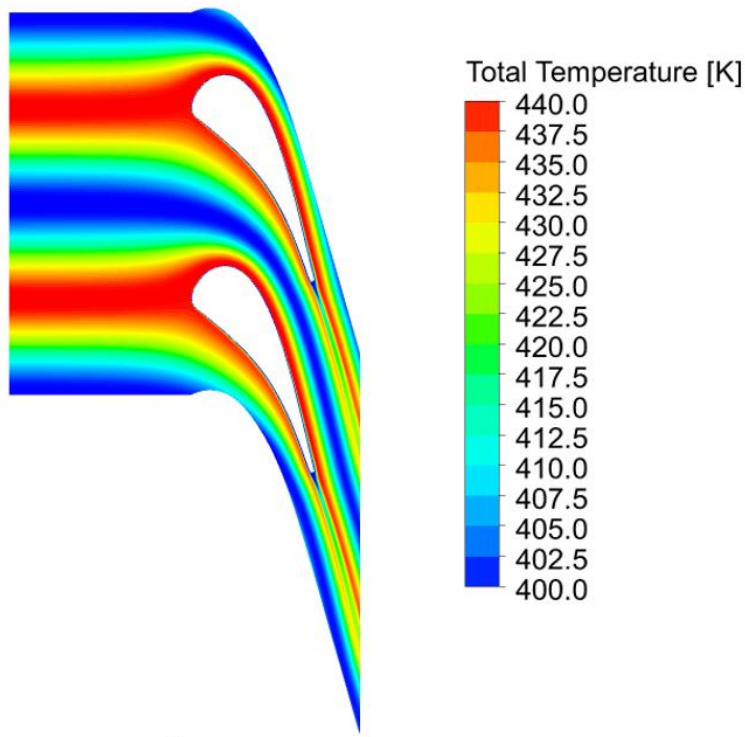

b) $\theta=270^{\circ}$ - Leading edge impingement

FIG. 15. Total temperature contours for two hot streak-vane relative circumferential position: a) Passage center, and b) Leading edge impingement.

To study the effects of relative clocking position between the hot streak and the turbine vane, both heat flux and heat transfer coefficient will be compared. It is noted that heat transfer coefficient for the hot streak investigation is now evaluated according to Equation 3 to account for the change in fluid driving temperature, which replaces the uniform inlet total temperature $T_{01}$ by the adiabatic wall temperature $T_{\mathrm{aw}}$. As a result, for each hot streak configuration, two computations need to be carried out at the adiabatic wall condition and at the isothermal wall condition to work out $T_{a w}$ and $q_{w}$ separately.

Figure 16 presents the area-averaged heat flux on the entire blade surface at different clocking positions and temperature distortion amplitudes in comparison with the uniform inlet case. It can be seen that circumferential positions affect significantly the heat flux strength. The sinusoidal distribution of the area-averaged heat flux can be attributed to the sinusoidal distribution of the imposed hot streak profile (e.g. Equation 9). The heat flux reaches its peak at $\theta=270^{\circ}$, where the blade is exposed to hot streak aligned with the leading edge. On the other hand, the heat flux reaches its trough at $\theta=90^{\circ}$, where the hot streak is aligned to the passage center. The amplitudes of heat flux peak and trough change proportionally to the amplitude of inlet temperature distortion. 


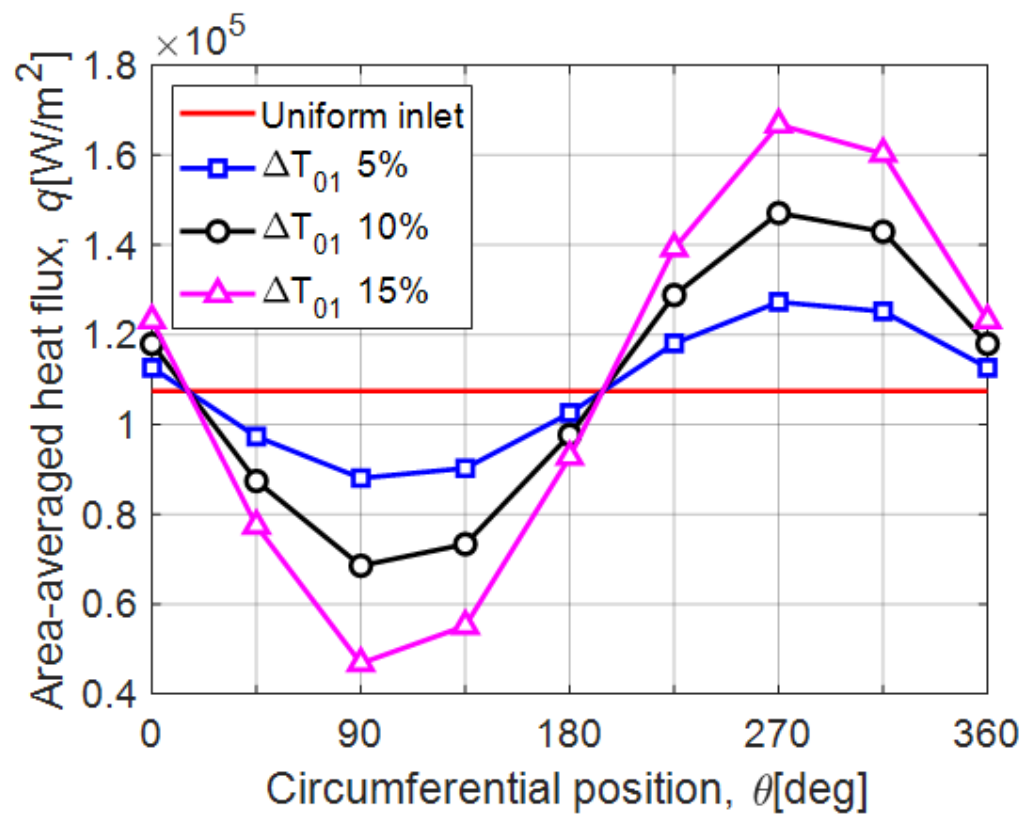

FIG. 16. Blade area-averaged heat flux at different circumferential positions and temperature distortion amplitudes.

In order to further explore the effects of hot streak temperature distortion amplitudes, the heat transfer coefficient at two most extreme circumferential positions will be compared. Figure 17 presents the heat transfer coefficient along the blade surfaces for different hot streak configurations. Although the area-averaged heat flux is remarkably different for the investigated hot streak configurations (see Figure 16), the surface heat transfer coefficient shows only minor changes except for the case with highest temperature distortion amplitude and hot streak aligned to passage center $\left(\theta=90^{0}, \Delta T_{01} 5 \%-\right.$ denoted by red solid line in Figure 17). At such hot streak conditions, the laminar-turbulent transition onset on the suction surface moves further downstream. It can also be observed that the difference between two clocking positions $\theta=90^{\circ}$ and $\theta=270^{\circ}$ increases as the temperature distortion increases. As the temperature distortion increases, the laminar-turbulent transition onset tends to move upstream for cases with the hot streak aligned to the leading edge $\left(\theta=270^{0}\right)$. On the other hand, the transition point tends to delay for cases with the hot streak aligned to the passage center $\left(\theta=90^{\circ}\right)$. This observation suggests that the hot streak clocking position has a significant effect on laminar-turbulence transition point and thus heat transfer coefficient at high distortion strength, while it has a minor effect at low distortion amplitude. 


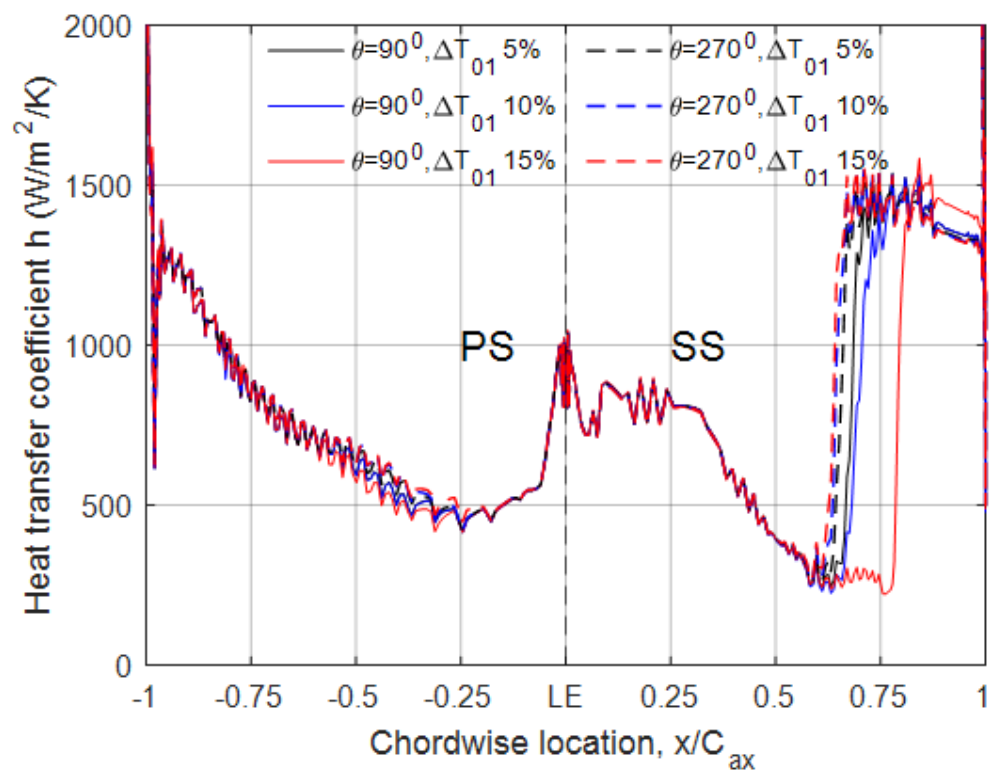

FIG. 17. Surface HTC for configurations with different temperature distortion amplitudes at two circumferential positions.

To enhance the understanding of temperature distortion strength effects, the turbulence intensity in the boundary layer region at vicinity to the blade suction surface is examined. The turbulence intensity $T u$ is calculated as in Equation 10:

$$
T u=\sqrt{\frac{2 k}{3}} / V
$$

where $k$ is the turbulence kinetic energy and $V$ is the mean velocity.

Only the suction surface is considered in this investigation due to its transition point susceptibility to temperature distortion, while the pressure surface shows insignificant responses to the temperature distortion strength and clocking positions. Figure 18 compares the turbulence intensity at both isothermal and adiabatic conditions. At first, it can be seen that the clocking position has negligible effects on the turbulence intensity for adiabatic computations, even at a high temperature distortion amplitude. In contrast, clocking position influences significantly the laminar-turbulent transition point for computations at the isothermal condition. For isothermal cases, the laminar-turbulent transition point moves further downstream as the temperature distortion strength increases for the hot streak passage center-alignment configuration. In other words, the transition onset is delayed as the fluid temperature impinged on the vane surface decreases. This is in agreement with some past research findings, in the way that the boundary layer is more stabilizing as the difference between the fluid and wall temperature decreases [18, 40]. In an extreme cases at the adiabatic condition, no heat transfer takes place across the blade surfaces, which represents the situation in which the difference between fluid and wall temperature vanishes. As a result, negligible differences in turbulence intensity prediction is observed among adiabatic computations. The recent findings show that aerodynamics and thermal coupling can be significant. Despite the wide belief that aerodynamics and heat transfer can be largely regarded as a one-way 
coupling such that aerodynamics would be affected marginally by the thermal conditions, the current results have shown that certain thermal conditions can influence the laminar-turbulent transition point. This implies that the two-way coupling of aerodynamics and heat transfer (conjugate heat transfer) should be taken into account in certain conditions, and the use of conventional technique could under-predict the transition point. Regarding the implication on turbomachinery research community, these results suggest that the experimental and numerical investigations of hot streak effects should be carried out near the engine-representative thermal conditions.

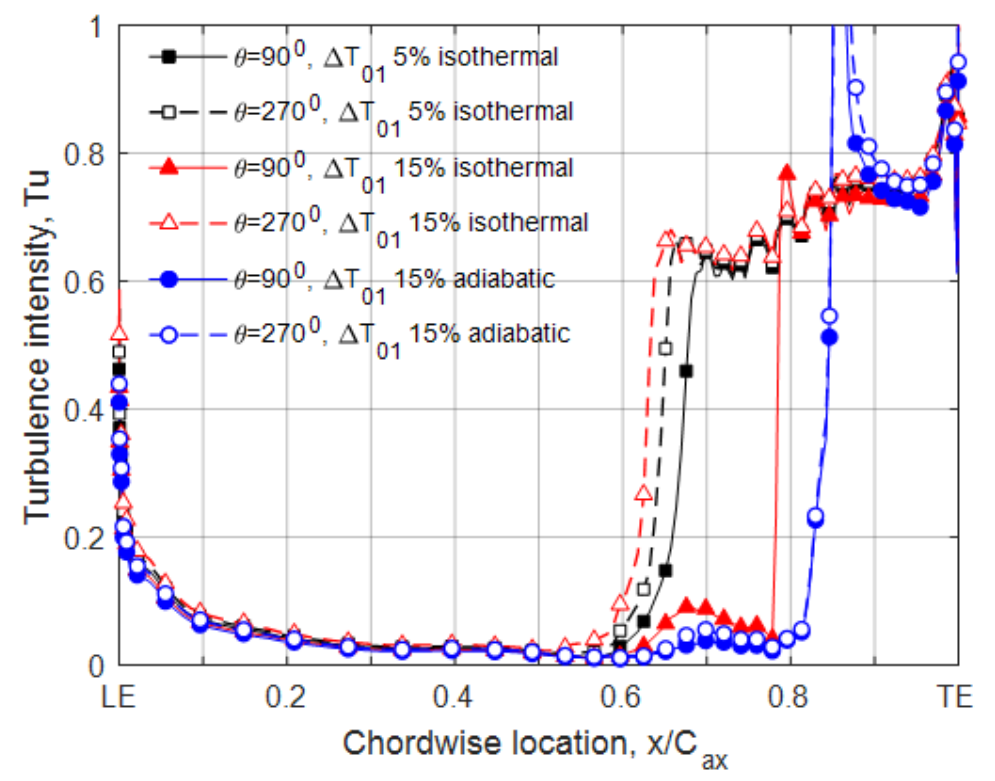

FIG. 18. Turbulence intensity distribution in the boundary layer region at vicinity to the suction surface for both isothermal and adiabatic conditions.

\section{FURTHER ANALYSIS ON THE UNSTEADINESS EFFECTS}

In previous computations, the unsteadiness effects are assumed to be small, thus enables the numerical investigations to be carried out efficiently with the steady flow model. In this section, the validity of such assumption is further analyzed by comparing the steady flow solution with the time-averaged solution resulting from ensemble averaging the unsteady flow model of test case MUR247.

Consider a two-dimensional problem for simplicity. The governing unsteady Navier-Stokes equations in Eq. 4-6 can be written in the integral form:

$$
\frac{\partial}{\partial t} \iint_{\Delta A} \boldsymbol{U} d A+\oint_{S}\left(\boldsymbol{F}-\boldsymbol{V}_{x}\right) d y+\oint_{S}\left(\boldsymbol{G}-\boldsymbol{V}_{y}\right) d x=0
$$

where 


$$
\begin{gathered}
\boldsymbol{U}=\left[\begin{array}{c}
\rho \\
\rho u \\
\rho v \\
\rho H
\end{array}\right], \boldsymbol{F}=\left[\begin{array}{c}
\rho u \\
\rho u u+P \\
\rho u v \\
\rho H u
\end{array}\right], \boldsymbol{G}=\left[\begin{array}{c}
\rho v \\
\rho u v+P \\
\rho v v+P \\
\rho H v
\end{array}\right] \\
\boldsymbol{V}_{x}=\left[\begin{array}{c}
0 \\
\tau_{x x} \\
\tau_{x y} \\
-q_{x}+u \tau_{x x}+v \tau_{x y}
\end{array}\right], \boldsymbol{V}_{y}=\left[\begin{array}{c}
0 \\
\tau_{x y} \\
\tau_{y y} \\
-q_{y}+u \tau_{x y}+v \tau_{y y}
\end{array}\right]
\end{gathered}
$$

The unsteady conservative variables can be further decomposed into a sum of a time-averaged component and an unsteady perturbation as in Equation 14. Note that the unsteady perturbation can be arisen due to both deterministic and stochastic disturbances.

$$
\boldsymbol{U}=\overline{\boldsymbol{U}}+\boldsymbol{U}^{\prime}
$$

If the expression in Equation 14 is substituted into the unsteady Navier-Stokes equations and then time averaged, the resultant time-averaged Navier-Stokes equations are:

$$
\oint_{S}\left(\overline{\boldsymbol{F}}-\overline{\boldsymbol{V}}_{x}\right) d y+\oint_{S}\left(\overline{\boldsymbol{G}}-\overline{\boldsymbol{V}}_{y}\right) d x=0
$$

where

$$
\begin{gathered}
\overline{\boldsymbol{F}}=\left[\begin{array}{c}
\overline{\rho u} \\
\bar{u} \overline{\rho u}+\bar{P}+\overline{(\rho u)^{\prime} u^{\prime}} \\
\overline{v \rho u}+\overline{(\rho u)^{\prime} v^{\prime}} \\
\bar{H} \overline{\rho u}+\overline{H^{\prime}(\rho u)^{\prime}}
\end{array}\right], \overline{\boldsymbol{G}}=\left[\begin{array}{c}
\overline{\rho v} \\
\bar{u} \overline{\rho v}+\bar{P}+\overline{(\rho v)^{\prime} u^{\prime}} \\
\overline{v \rho v}+\overline{(\rho v)^{\prime} v^{\prime}} \\
\bar{H} \overline{\rho v}+\overline{H^{\prime}(\rho v)^{\prime}}
\end{array}\right] \\
\overline{\boldsymbol{V}}_{x}=\left[\begin{array}{c}
0 \\
\bar{\tau}_{x x} \\
\bar{\tau}_{x y} \\
-\bar{q}_{x}+\bar{u} \bar{\tau}_{x x}+\bar{v} \bar{\tau}_{x y}+\overline{u^{\prime} \tau_{x x}^{\prime}}+\overline{v^{\prime} \tau_{x y}^{\prime}}
\end{array}\right], \overline{\boldsymbol{V}}_{y}=\left[\begin{array}{c}
\bar{\tau}_{y y} \\
-\bar{q}_{y}+\bar{u} \bar{\tau}_{x y}+\bar{v} \bar{\tau}_{y y}+\overline{u^{\prime} \tau_{x y}^{\prime}}+\overline{v^{\prime} \tau_{y y}^{\prime}}
\end{array}\right]
\end{gathered}
$$

Comparing the steady form of the unsteady governing equations (with no temporal term) and the set of time-averaged governing equations in Eq. 16-17, one can notice the existence of extra terms in the time-averaged equations (i.e. the correlation of fluctuating variables). These extra terms have a similar form to the Reynolds stress term (also denoted as the unsteady stress term [41]). The terms exist due to the nonlinearity effects of turbulence flow, thus their magnitudes are indicators of the nonlinear severity.

Figure 19 presents the discrepancy in the heat transfer coefficient prediction in the steady flow and time-averaged solution, $\Delta h=\left|H T C_{\text {steady }}-H T C_{\text {time-averaged }}\right|$. With the large portion of the blade surface has a very low $\Delta h$, it is clear that the majority of the blade surface has an equivalent heat transfer coefficient prediction between the two solutions. There are two zones with distinctively higher heat transfer coefficient prediction discrepancy: rear part of the suction surface and the trailing edge. The rear part of the suction surface has a moderate discrepancy magnitude. Recalling the previous discussion on this part, 
it is observed that this part is in the region of the laminar-turbulent transition. Thus, the inherently unsteady behavior of the laminar-turbulent transition induces a moderate change in the time-averaged heat transfer coefficient due to its nonlinearity. On the other hand, a much more dramatic change in heat transfer coefficient is observed in the trailing edge region on both the pressure and suction surfaces. Even up to $50 \%$ of the heat transfer coefficient change is spotted in this region on the suction surface. Similarly to the case of the laminar-turbulent transition region, the change in heat transfer coefficient between two solutions stems from the nonlinearity of the local flow field. An extremely high change in terms of heat transfer coefficient magnitude is subject to the highly local turbulent flow field as well as the contributions from the deterministic disturbances due to the vane trailing edge wake and vortex shedding. Although the local heat transfer coefficient between the steady and timeaveraged solutions is remarkably different at the trailing edge region, the total area-averaged heat transfer coefficient on the entire blade surface changes by only $2 \%$. This is due to the small area of the blade surface that is subject to strong flow nonlinearity (the laminar-turbulent transition and the trailing edge region). As a result, it can be deduced that the unsteadiness effects shall be take into account if the local heat transfer distribution is of interest, especially if the flow field is highly nonlinear.

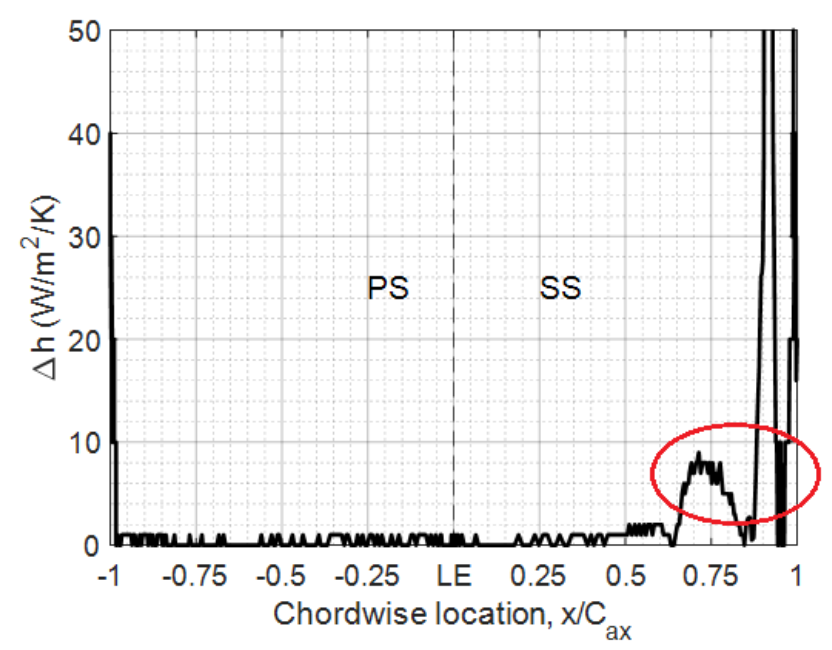

FIG. 19. Difference in heat transfer coefficient prediction between the steady flow and time-averaged solutions.

\section{CONCLUSIONS}

The present study has successfully validated the modeling techniques in heat transfer prediction as applied to the nozzle guide vane in the high-pressure turbine blade row. Comparisons with the available experimental data for both aerodynamic and heat transfer test cases yield a satisfactory agreement. With the heat transfer test case, it has been shown that the laminarturbulent transition is paramount to the suction surface heat transfer coefficient prediction. Thus, the use of the transitional turbulence model is recommended, at least for this test case flow conditions. If the fully turbulent model is used, the heat 
transfer coefficient is over-predicted on both surfaces. On the other hand, heat transfer coefficient is under-predicted at the rear part of the suction surface if the fully laminar model is used due to the formation of a large open-type flow separation.

The focus of the present work is on the impact of the heat transfer performance predictability with uncertainties in the inflow boundary conditions. At first, the inlet turbulence intensity is a subject of interest. The heat transfer coefficient on the pressure surface increases significantly, and a quantitative prediction of CFD modeling can be expected. On the suction surface, the prediction of transition location has a good agreement. In addition, it has been observed that the turbulence intensity decays rapidly from the inlet station to the vane leading edge in the computational model in agreement with the analytical prediction, whereas the rate of decay in the experiment is not known. The rapid decay of the turbulence intensity in the computational model would induce a rather small but erroneous change of heat transfer coefficient prediction in configurations with increasing inlet turbulence intensity. It has been found that a significant improvement of the computational prediction can be achieved if an artificially high inflow turbulence intensity is employed in the numerical model to compensate for the high decay rate.

In the second sensitivity study, the inlet endwall boundary layer thickness is varied by imposing a corresponding radial total pressure profile. The heat transfer augmentation area is in line with the complicated vortical flow structures formed in a threedimensional cascade. The local heat transfer coefficient changes more significantly in the near-endwall region. However, on the entire blade surface, this near-endwall region has a limited influence on the total area-averaged heat transfer coefficient. On the other hand, a dramatic increase of heat load on the endwall surface with increasing inlet endwall boundary layer thickness is observed.

The last sensitivity study involves the non-uniform inlet temperature profile that one would expect to see downstream the combustor. The heat flux distribution has been shown to be affected greatly by both the clocking position and distortion amplitude. In contrast, the heat transfer coefficient remains largely unaffected except for the configuration with a small difference between the fluid driving temperature and wall temperature (i.e. near-adiabatic thermal conditions). The heat flux in isothermal cases contributes to the destabilizing effect of the boundary layer on the suction surface. The findings have been consolidated by examination of earlier transition onset in the high heat flux configurations. The results suggest that conjugate heat transfer might be necessary in a few cases where the aero-thermal coupling is sufficiently strong. The results also point out that experimental and numerical hot streak investigation shall be conducted near the engine-representative thermal conditions.

Finally, further analysis has been carried out to examine the steady flow assumption used in the current computational model. By the comparison between the steady form of the unsteady Navier-Stokes equations and the set of time-averaged equations, the generation of extra terms has been noted. These terms have a similar form to the Reynolds stress term, which 
arise due to the nonlinearity characteristics of the flow field. The heat transfer coefficient prediction between the steady and time-averaged solution have been compared to reveal two zones with high discrepancy: the rear part of the suction surface and the trailing edge. The prediction discrepancy in these areas is attributed to the nonlinear behavior of the laminar-turbulent transition as well as the highly turbulent flow and vane trailing edge vortex shedding. Although local heat transfer coefficient is remarkably different in a few regions, the total area-averaged heat transfer coefficient prediction is almost comparable between the steady and time-averaged computations.

\section{NOMENCLATURE}

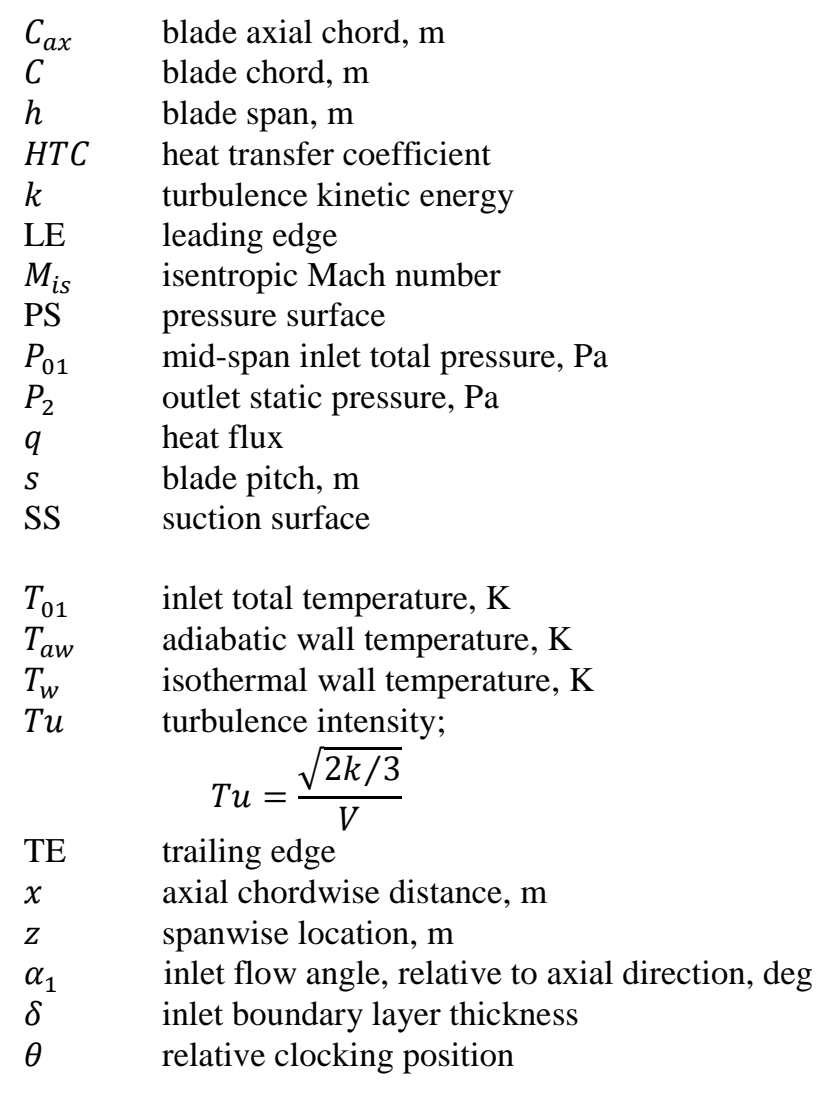

\section{REFERENCES}

${ }^{1}$ Wheeler, A. P., Sandberg, R. D., Sandham, N. D., Pichler, R., Michelassi, V. and Laskowski, G., "Direct numerical simulations of a high-pressure turbine vane," J. Turbomach. 138 (7), 071003 (2016).

${ }^{2}$ Michelassi, V., Chen, L. W., Pichler, R. and Sandberg, R. D., "Compressible direct numerical simulation of low-pressure turbines," J. Turbomach. 137 (7), 071005 (2015).

${ }^{3}$ Fang, J., Gao, Y., Liu, Y., Lu, L., Yao, Y. and Le Ribault, C., "Direct numerical simulation of a tip-leakage flow in a planar duct with a longitudinal slit," Phys. Fluids 31 (12), 125108 (2019).

${ }^{4}$ Fang, X., Yang, Z., Wang, B. C., Tachie, M. F. and Bergstrom, D. J., "Large-eddy simulation of turbulent flow and structures in a square duct roughened with perpendicular and V-shaped ribs," Phys. Fluids 29 (6), 065110 (2017).

${ }^{5}$ Xun, Q. Q. and Wang, B. C., "Hybrid RANS/LES of turbulent flow in a rotating rib-roughened channel," Phys. Fluids 28 (7), 075101 (2016).

${ }^{6}$ Thole, K. A. and Knost, D. G., "Heat transfer and film-cooling for the endwall of a first stage turbine vane," Int. J. Heat Mass Transf 48 (25-26), 5255-5269 (2005). 
${ }^{7}$ Hada, S., Takeishi, K., Oda, Y., Mori, S. and Nuta, Y., "The effect of leading edge diameter on the horse shoe vortex and endwall heat transfer," ASME Paper No. GT2008-50892, 2008.

${ }^{8}$ Arts, T., Duboue, J. M. and Rollin, G., "Aerothermal performance measurements and analysis of a two-dimensional high turning rotor blade," J. Turbomach. 120 (3), 494-499 (1998).

${ }^{9}$ Stripf, M., Schulz, A. and Wittig, S., "Surface roughness effects on external heat transfer of a HP turbine vane," J. Turbomach. 127 (1), 200-208 (2005).

${ }^{10}$ Denton J. D., "Some limitations of turbomachinery CFD," ASME Paper No. GT2010-22540, 2010.

${ }^{11}$ Phan, H. M. and He, L., "Validation studies of linear oscillating compressor cascade and use of influence coefficient method," J. Turbomach. (2019). https://doi.org/10.1115/1.4045657

${ }^{12}$ Zhang, Q., He, L. and Rawlinson, A., "Effects of inlet turbulence and end-wall boundary layer on aerothermal performance of a transonic turbine blade tip," J. Eng. Gas Turb. Power 136 (5), 052603 (2014).

${ }^{13} \mathrm{Qu}, \mathrm{X}$., Zhang, Y., Lu, X. and Zhu, J., "Unsteady effects of periodic wake passing frequency on aerodynamic performance of ultra-high-lift low pressure turbine cascades," Phys. Fluids 31 (9), 094102 (2019).

${ }^{14}$ Jia, L., Zou, T., Zhu, Y. and Lee, C., "Rotor boundary layer development with inlet guide vane (IGV) wake impingement," Phys. Fluids 30 (4), 040911 (2018).

${ }^{15}$ Choi, J., Teng, S., Han, J. C. and Ladeinde, F., "Effect of free-stream turbulence on turbine blade heat transfer and pressure coefficients in low Reynolds number flows," Int. J. Heat Mass Transf 47 (14-16), 3441-3452 (2004).

${ }^{16}$ Wang, Z., Wang, D., Liu, Z. and Feng, Z., "Numerical analysis on effects of inlet pressure and temperature non-uniformities on aero-thermal performance of a HP turbine," Int. J. Heat Mass Transf 104, 83-97 (2017).

${ }^{17} \mathrm{He}$, L., Menshikova, V. and Haller, B. R., "Effect of hot-streak counts on turbine blade heat load and forcing," J. Propul. Power 23 (6), 1235-1241 (2007).

${ }^{18}$ Wang, Z., Liu, Z. and Feng, Z., "Influence of mainstream turbulence intensity on heat transfer characteristics of a high pressure turbine stage with inlet hot streak," J. Turbomach. 138 (4), 041005 (2015).

${ }^{19}$ Rahim, A. and He, L., "Rotor blade heat transfer of high pressure turbine stage under inlet hot-streak and swirl," J. Eng. Gas Turb. Power 137 (6), 062601 (2015).

${ }^{20}$ Rahim, A., Khanal, B., He, L. and Romero, E., "Effect of nozzle guide vane lean under influence of inlet temperature traverse," J. Turbomach. 136 (7), 071002 (2014).

${ }^{21}$ Khanal, B., He, L., Northall, J. and Adami, P., "Analysis of radial migration of hot-streak in swirling flow through highpressure turbine stage," J. Turbomach. 135 (4), 041005 (2013).

${ }^{22}$ Ong, J. and Miller, R. J., "Hot streak and vane coolant migration in a downstream rotor," J. Turbomach. 134 (5), 051002 (2012).

${ }^{23}$ Feng, Z., Liu, Z., Shi, Y. and Wang, Z., "Effects of hot streak and airfoil clocking on heat transfer and aerodynamic characteristics in gas turbine," J. Turbomach. 138 (2), 021002 (2015).

${ }^{24}$ Takeishi, K., Matsuura, M., Aoki, S. and Sato, T., "An experimental study of heat transfer and film cooling on low aspect ratio turbine nozzles," J. Turbomach. 112 (3), 488-496 (1990).

${ }^{25}$ Friedrichs, S., "Endwall film-cooling in axial flow turbines," Ph.D. thesis, University of Cambridge, Cambridge, United Kingdom, 1997.

${ }^{26}$ Lynch, S. P. and Thole, K. A., "The effect of combustor-turbine interface gap leakage on the endwall heat transfer for a nozzle guide vane," J. Turbomach. 130 (4), 041019 (2008).

${ }^{27}$ Lee, K. D. and Kim, K. Y., "Shape optimization of a fan-shaped hole to enhance film-cooling effectiveness," Int. J. Heat Mass Transf 53 (15-16), 2996-3005 (2010).

${ }^{28}$ Arts, T. and De Rouvroit, M. L., "Aero-thermal performance of a two-dimensional highly loaded transonic turbine nozzle guide vane: a test case for inviscid and viscous flow computations," J. Turbomach. 114 (1), 147-157 (1992).

${ }^{29}$ Arts, T., Lambertderouvroit, M. and Rutheford, A. W., "Aero-thermal investigation of a highly loaded transonic linear turbine guide vane cascade. A test case for inviscid flow and viscous flow computations," NASA STI/Recon Technical Report N 91 (1992).

${ }^{30}$ Menter, F. R., Langtry, R. B., Likki, S. R., Suzen, Y. B., Huang, P. G. and Völker, S., "A correlation-based transition model using local variables-Part I: Model formulation," J. Turbomach. 128 (3), 413-422 (2006).

${ }^{31}$ Langtry, R. B., Menter, F. R., Likki, S. R., Suzen ,Y. B., Huang, P. G. and Völker, S., "A correlation-based transition model using local variables-Part II: Test cases and industrial applications," J. Turbomach. 128 (3), 423-434 (2006).

${ }^{32}$ Dong, H., Xia, T., Chen, L., Liu, S., Cui, Y. D., Khoo, B. C. and Zhao, A., "Study on flow separation and transition of the airfoil in low Reynolds number," Phys. Fluids 31 (10), 103601 (2019).

${ }^{33}$ Fawcett, R. J., Wheeler, A. P., He, L. and Taylor, R., "Experimental investigation into the impact of crossflow on the coherent unsteadiness within film cooling flows," Int. J. Heat Fluid Fl. 40, 32-42 (2013).

${ }^{34}$ Fawcett, R. J., Wheeler, A. P., He, L. and Taylor, R., "Experimental investigation into unsteady effects on film cooling," J. Turbomach. 134 (2), 021015 (2012).

${ }^{35}$ Zhou, W., Chen, H., Liu, Y. and Peng, D., "Unsteady analysis of adiabatic film cooling effectiveness for discrete hole with oscillating mainstream flow," Phys. Fluids 30 (12), 127103 (2018). 
${ }^{36}$ Vagnoli, S., "Assessment of advanced numerical methods for the aero-thermal investigation of combustor-turbine interactions," Ph.D. thesis, University of Florence, Florence, Italy, 2016.

${ }^{37}$ Langtry, R. B., "A correlation-based transition model using local variables for unstructured parallelized CFD codes," Ph.D. thesis, University of Stuttgart, Stuttgart, Germany, 2006.

${ }^{38}$ Luo, J. and Razinsky, E. H., "Prediction of heat transfer and flow transition on transonic turbine airfoils under high free stream turbulence," ASME Paper No. GT2008-50868, 2008.

${ }^{39}$ Segui, L., Gicquel, L., Duchaine, F. and de Laborderie, J., "LES of the LS89 cascade: influence of inflow turbulence on the flow predictions," ETC Paper No. ETC2017-159, 2017.

${ }^{40}$ Rubini, R., Maffulli, R. and Arts, T., "Effect of the gas to wall temperature ratio on the bypass transition," J. Turbomach. (2019). https://doi.org/10.1115/1.4043782

${ }^{41}$ He, L. and Ning, W., "Efficient approach for analysis of unsteady viscous flows in turbomachines," AIAA J. 36 (11), $2005-$ 2012 (1998). 